\title{
Are morbidity and mortality conferences becoming a lost art?
}

The views expressed in this editorial are those of the author and do not necessarily reflect the position of the Canadian Medical Association or its subsidiaries.

urgical morbidity and mortality $(M \& M)$ conferences have a history that is both deep and central to many of the core principles we hold dear to our profession. More specifically, well-run M\&M conferences employ a peer review process that analyzes adverse patient outcomes on multiple levels. This evaluation includes a discussion of the mechanics of the negative event itself; suggestion of modifications in behaviour, analysis, decision-making and/or technique; and finally system improvements to prevent similar errors from occurring in the future.

The origin of $M \& M$ conferences possessed a challenging ignition in 1904 when Ernest Codman famously suggested that surgeon competence must be evaluated and reported in a structured and repetitive manner. ${ }^{1}$ While this altruistic beginning led to his firing from the Massachusetts General Hospital, Codman's ideas were relatively quick to be parlayed into the American College of Surgeons case reporting system for adverse patient outcomes in 1916. ${ }^{1}$ It could be argued that Codman's concept reached its zenith in 1983 when the Accreditation Council for Graduate Medical Education (the American equivalent of our Royal College of Physicians and Surgeons of Canada) mandated the presence of weekly $M \& M$ conferences to achieve and maintain accreditation for all surgical residency training programs. ${ }^{1}$

As many of us travel throughout the world to engage in postresidency surgical fellowships, visit our colleagues' institutions, pursue mid-career academic sabbaticals, and/or deliver formal visiting professorships, it becomes quickly apparent that the precise delivery of Codman's M\&M conferences varies dramatically from site to site. ${ }^{2}$ In some institutions, $M \& M$ conferences are city-wide endeavours that include multiple hospitals sharing their adverse patient outcomes, whereas in others, it remains limited to a small surgical subspecialty group discussing patients and diagnoses specific to a very narrow slice of medicine. In a few institutions, the discussion is focused on CanMEDS learning objectives that are indirect to the event itself. Irrespective of the delivery, however, an environment that is nonpunitive to the individual surgeon, in conjunction with a focus on quality improvement in patient care (i.e., future prevention of a similar error) is essential.
It has been increasingly evident in recent years that the central importance of weekly $M \& M$ conferences (and the associated quality-improvement opportunities) seems to be less revered and accepted among today's generation of surgeons. While the postulates for this observation are fascinating, a recurring theme now includes the impact of "big data" quality improvement opportunities such as the National Surgical Quality Improvement Program (NSQIP). More specifically, an increasingly popular narrative is that NSQIP and its comparators have essentially replaced the need for formal small-group $M \& M$ conferences. While these large and powerful quality-improvement platforms are rapidly becoming ingrained within our surgical practices for everything from trauma to emergency general surgery to highly selected subspecialty work, the local wisdom and experience available in a typical $M \& M$ conference remains critical to both surgeon improvement and peak performance. As a result, it seems that the future of Codman's call for the continued evaluation of competence must involve both comparisons of surgeon performance to larger groups of colleagues at the individual and program levels (i.e., via big data), as well as the incorporation of local expertise and sage advice in the form of collegial and real-time discussion at a formal M\&M conference. ${ }^{3}$ In other words, never has the role of the M\&M conference been so important to the mission of continued quality improvement in patient care at each of our institutions and hospital systems.

\author{
Chad G. Ball, MD \\ Coeditor, Canadian fournal of Surgery
}

Competing interests: None declared.

DOI: $10.1503 /$ cjs.004119

\section{References}

1. Gregor A, Taylor D. Morbidity and mortality conference: its purpose reclaimed and grounded in theory. Teach Learn Med 2016; 28:439-47.

2. Campbell W. Surgical morbidity and mortality meetings. Ann R Coll Surg Engl 1988;70:363-5.

3. McNamara DA, Hall HM, Hardin EA. Rethinking the modern cardiology morbidity and mortality conference: harmonizing quality improvement and education. $\mathcal{F}$ Am Coll Cardiol. 2019;73:868-72. 